\title{
Bag representation for composite degrees of freedom in lattice gauge theories with fermions
}

\author{
Carlotta Marchis * $\dagger$ \\ University of Graz, Institute of Physics, 8010 Graz, Austria \\ E-mail: carlotta.marchis@uni-graz.at

\section{Christof Gattringer} \\ University of Graz, Institute of Physics, 8010 Graz, Austria \\ E-mail: christof.gattringer@uni-graz.at

\section{Oliver Orasch} \\ University of Graz, Institute of Physics, 8010 Graz, Austria \\ E-mail: oliver.orasch@uni-graz.at
}

\begin{abstract}
We explore new representations for lattice gauge theories with fermions, where the space-time lattice is divided into dynamically fluctuating regions, inside which different types of degrees of freedom are used in the path integral. The first kind of regions is a union of so-called bags, in which the dynamics is described by the free propagation of composite degrees of freedom of the original fermions. In the second region, called complementary domain, configurations of the remaining interacting degrees of freedom are used to describe the dynamics. We work out the bag representation for the gauge groups $\mathrm{SU}(2)$ and $\mathrm{SU}(3)$ and address the nature of the strong coupling effective degrees of freedom, which are fermions for $\mathrm{SU}(3)$ and bosons for $\mathrm{SU}(2)$. We discuss first steps towards a numerical simulation of the bag representations.
\end{abstract}

The 36th Annual International Symposium on Lattice Field Theory - LATTICE2018

22-28 July, 2018

Michigan State University, East Lansing, Michigan, USA.

\footnotetext{
${ }^{*}$ Speaker.

$\dagger$ This work is supported by the Austrian Science Fund FWF, grant I 2886-N27 and the FWF DK W 1203, "Hadrons in Vacuum, Nuclei and Stars".
} 


\section{Introduction}

In recent years an important development in lattice field theories was the construction of worldline and worldsheet representations (see, e.g., the reviews at the annual lattice conferences [1]). Often the motivation are lattice field theories at finite density where in some cases the complex action problem may be overcome by a suitable worldline/worldsheet representation. For systems with fermions, such as QCD, the situation is more complicated due to the Grassmann nature of the fermions and additional signs from the Clifford algebra. However, for strong coupling worldline representations have been studied since the early days of lattice field theory [2]. This idea has been revisited recently and interesting results were obtained for strong coupling QCD [3, 4]. Also beyond strong coupling suggestions for a fully dualized version of lattice QCD [5] were presented.

Also for purely fermionic lattice field theories worldline representations play an important role. In some cases it is possible to develop a representation in terms of so-called fermion bags. Fermion bags are space-time domains on the lattice where the dynamics is essentially given by free fermions which inside the bag can be described by a fermion determinant. In between the bags the fermionic Grassmann integral is saturated with the interaction terms [6]. For many interesting systems fermion bag representations were applied as a powerful tool for numerical simulations [7].

In this contribution we discuss further developments based on [4], where a so-called baryon bag representation was constructed for strong coupling lattice QCD. Although the technical aspects of a simulation with baryon bags are similar to the fermion bag concept, the underlying physical picture is different: Inside a baryon bag three strongly coupled quarks propagate jointly as a baryon which is described as a free composite fermion (whereas inside a conventional fermion bag the fundamental fermions of the theory propagate as free fermions). The physics inside the baryon bag is described by a free fermion determinant, while outside the baryon bags the dynamics is governed by quark and diquark monomer and dimer terms. Here we report on developments towards an efficient simulation of the monomers and dimers and also discuss the case of strong coupling $\mathrm{SU}(2)$, where the degrees of freedom inside the bags are nilpotent bosons, that are described by bag permanents instead of the bag determinants that appear for fermionic effective degrees of freedom.

\section{Factorization of strong coupling QCD}

We begin our presentation with a brief summary of the baryon bag formulation [4] of strong coupling QCD. We use one flavor of staggered fermions, where the action is given by

$$
S_{F}[\bar{\psi}, \psi, U]=\sum_{x}\left(2 m \bar{\psi}_{x} \psi_{x}+\sum_{v} \gamma_{x, v}\left[\bar{\psi}_{x} U_{x, v} \psi_{x+\hat{v}}-\bar{\psi}_{x+\hat{v}} U_{x, v}^{\dagger} \psi_{x}\right]\right) .
$$

$\psi_{x}$ and $\bar{\psi}_{x}$ are Grassmann variables with 3 color components and by $\gamma_{x, v}$ we denote the staggered sign factors $\gamma_{x, 1}=1, \gamma_{x, 2}=(-1)^{x_{1}}, \gamma_{x, 3}=(-1)^{x_{1}+x_{2}}$ and $\gamma_{x, 4}=(-1)^{x_{1}+x_{2}+x_{3}}$. The gauge fields are coupled via the link variables $U_{x, v} \in \mathrm{SU}(3)$. At strong coupling the partition sum is given by

$Z=\int D[\bar{\psi}, \psi] \int D[U] e^{S_{F}[\bar{\psi}, \psi, U]}=\int D[\bar{\psi}, \psi] \prod_{x} e^{2 m \bar{\psi}_{x} \psi_{x}} \int D[U] \prod_{x, \nu} e^{\gamma_{x, \nu} \bar{\psi}_{x} U_{x, \nu} \psi_{x+\hat{v}}} e^{-\gamma_{x, \nu} \bar{\psi}_{x+\hat{\nu}} U_{x, \nu}^{\dagger} \psi_{x}}$ 
where in the second step we have written the sums in the action as products of the individual Boltzmann factors. The exponentials of the nearest neighbor terms can be expanded into power series that terminate after the third order, since for gauge group SU(3) we have only 3 independent (pairs of) Grassmann variables per site (for notational convenience we here drop the space-time indices and use matrix-vector notation for the color indices)

$$
e^{\gamma \bar{\psi} U \psi}=1+\gamma \bar{\psi} U \psi+\frac{(\bar{\psi} U \psi)^{2}}{2 !}+\frac{(\gamma \bar{\psi} U \psi)^{3}}{3 !}=\left[1+\gamma \frac{(\bar{\psi} U \psi)^{3}}{3 !}\right]\left[1+\gamma \bar{\psi} U \psi+\frac{(\bar{\psi} U \psi)^{2}}{2 !}\right] .
$$

In the second step we have pulled out the highest monomial and the product of the two square brackets reproduces the previous expression due to nilpotency. It is easy to see that the 3rd order term is independent of the gauge link $U:(\bar{\psi} U \psi)^{3}=\left(\bar{\psi}_{a} U_{a b} \psi_{b}\right)^{3}=3 ! \bar{\psi}_{3} \bar{\psi}_{2} \bar{\psi}_{1} \psi_{1} \psi_{2} \psi_{3} \operatorname{det} U \equiv$ $3 ! \bar{B} B$, where in the last step we have used $\operatorname{det} U=1$ and introduced the baryon fields $B_{x}, \bar{B}_{x}$ defined as $B_{x}=\psi_{x, 1} \psi_{x, 2} \psi_{x, 3}, \bar{B}_{x}=\bar{\psi}_{x, 3} \bar{\psi}_{x, 2} \bar{\psi}_{x, 1}$. Thus we obtain

$$
e^{\gamma \bar{\psi} U \psi}=e^{\gamma \bar{B} B} \sum_{d=0}^{2} \frac{(\gamma \bar{\psi} U \psi)^{d}}{d !}, e^{-\gamma \bar{\psi} U^{\dagger} \psi}=e^{-\gamma \bar{B} B} \sum_{\bar{d}=0}^{2} \frac{\left(-\gamma \bar{\psi} U^{\dagger} \psi\right)^{\bar{d}}}{\bar{d} !}, e^{2 m \bar{\psi} \psi}=e^{(2 m)^{3} \bar{B} B} \sum_{s=0}^{2} \frac{(2 m \bar{\psi} \psi)^{s}}{s !},
$$

where we have repeated the steps for the forward hopping term also for the backward hopping and mass terms. We stress again, that all contributions that we have expressed in terms of the baryon fields $B_{x}$ and $\bar{B}_{x}$ are independent of the gauge fields. Using the results (2.4) we find for the partition sum $Z=\int D[\bar{\psi}, \psi] e^{S_{B}[\bar{B}, B]} W[\bar{\psi}, \psi]$. We have factorized the contributions of the baryons and organized them in the baryon action (we re-inserted all space-time indices)

$$
S_{B}[\bar{B}, B]=\sum_{x}\left(2 M \bar{B}_{x} B_{x}+\sum_{v} \gamma_{x, v}\left[\bar{B}_{x} B_{x+\hat{v}}-\bar{B}_{x+\hat{v}} B_{x}\right]\right),
$$

which has the form of a free staggered action for the baryon fields $B_{x}$ and $\bar{B}_{x}$ with mass $M=4 m^{3}$.

\section{Strong coupling integrals}

The remaining, non-baryonic terms depend on the gauge fields and we have collected them in

$$
W[\bar{\psi}, \psi]=\prod_{x} \sum_{s_{x}} \frac{\left(2 m \bar{\psi}_{x} \psi_{x}\right)^{s_{x}}}{s_{x} !} \prod_{x, v} \sum_{d_{x, v}, \bar{d}_{x, v}} \frac{\left(\gamma_{x, v}\right)^{d_{x, v}+\bar{d}_{x, v}}}{d_{x, v} ! \bar{d}_{x, v} !} \int D[U]\left(\bar{\psi}_{x} U_{x, v} \psi_{x+\hat{v}}\right)^{d_{x, v}}\left(-\bar{\psi}_{x+\hat{v}} U_{x, v}^{\dagger} \psi_{x}\right)^{\bar{d}_{x, v}}
$$

The integrals over the gauge links project to color singlet combinations. These are obtained either by the trivial choice $d_{x, v}=\bar{d}_{x, v}=0$, the quark dimer term $d_{x, v}=\bar{d}_{x, v}=1$ or the diaquark dimer term $d_{x, v}=\bar{d}_{x, v}=2$. The corresponding integrals are well known, see, e.g., [8], and we find [4]

$$
Z=\int D[\bar{\psi}, \psi] e^{S_{B}[\bar{B}, B]} \prod_{x} \sum_{s_{x}=0}^{2} \frac{\left(2 m \bar{\psi}_{x} \psi_{x}\right)^{s_{x}}}{s_{x} !} \prod_{x, v} \sum_{d_{x, v}=0}^{2} \frac{\left(3-d_{x, v}\right) !}{6 d_{x, v} !}\left(\bar{\psi}_{x} \psi_{x} \bar{\psi}_{x+\hat{v}} \psi_{x+\hat{v}}\right)^{d_{x, v}}
$$

The final step is to saturate the Grassmann integral. The first possibility is to saturate the Grassmann integral using the baryon terms. Since the baryon fields $B_{x}$ and $\bar{B}_{x}$ contain all three colors, the 
baryon terms completely saturate the Grassmann integral on the sites where we use them. The fact that there are no remaining quark link terms at strong coupling also implies that the regions where we saturate the Grassmann integral with the baryon terms do not mix with the other sites where we use the terms from $W[\bar{\psi}, \psi]$. We now refer to a connected area of space-time where we use the baryon terms for saturating the Grassmann integral as a baryon bag $\mathscr{B}_{i}$. By $\mathscr{B}$ we denote the union of all bags. Since the $B_{x}$ and $\bar{B}_{x}$ inherit the Grassmann properties of the underlying quark fields [4], the contribution of the baryons inside the bag $\mathscr{B}_{i}$ is given by the determinant $\operatorname{det} D^{(i)}$ of the free staggered Dirac operator $D^{(i)}$ from the baryon action (2.5), but restricted to the sites of $\mathscr{B}_{i}$.

In the complementary domain $\overline{\mathscr{B}}$ we saturate the Grassmann integral by combining quark monomer $\left(s_{x}=1\right)$ and diquark monomer terms $\left(s_{x}=2\right)$ on a single site, with quark dimers $\left(d_{x, v}=1\right)$ and diquark dimers $\left(d_{x, v}=2\right)$ on links. Note that since the Grassmann variables are already in the correct order no negative signs remain. We summarize the baryon bag form as [4],

$$
Z=\sum_{\{\mathscr{B}\}} \prod_{i} \operatorname{det} D^{(i)} \sum_{\{s, d \| \overline{\mathscr{B}}\}}(2 m)^{\sum_{x} s_{x}}\left(\frac{1}{3}\right)^{\sum_{x, v}\left[\delta_{d_{x, v}, 1}+\delta_{d_{x, v}, 2}\right]} .
$$

The sum runs over all possible ways of decomposing the lattice into baryon bags $\mathscr{B}_{i}$ and a complementary domain $\overline{\mathscr{B}}$. For each bag $\mathscr{B}_{i}$ we collect as a factor the determinant $\operatorname{det} D^{(i)}$ of the Dirac operator $D^{(i)}$ in (2.5) restricted to $\mathscr{B}_{i}$. In the complementary domain we saturate the Grassmann integral with with quark monomers $\left(s_{x}=1\right)$, diquark monomers $\left(s_{x}=2\right)$, quark dimers $\left(d_{x, v}=1\right)$ and diquark dimers $\left(d_{x, v}=2\right)$, and by the second sum we denote the sum over all admissible monomer and dimer configurations compatible with a given complementary domain $\overline{\mathscr{B}}$. Quark and diquark monomers come with a factor $(2 m)^{s_{x}}$, quark and diquark dimers both with a factor of $1 / 3$.

\section{Towards an efficient update strategy}

We begin the discussion of possible update strategies for a numerical simulation of the baryon bag representation with the complementary domain $\overline{\mathscr{B}}$. Here we have to occupy each site of the lattice with quark and diquark monomers or with the endpoints of quark and diquark dimers. More specifically each site of the lattice has three possible colors where we can attach the corresponding elements. In order to illustrate this, in Fig. 1 we show a chain of neighboring sites $x_{1}, x_{2} \ldots x_{6}$. For every site we use three layers that correspond to the three colors. A quark monomer is represented by a single square and a diquark monomer by a rectangle that covers two layers. Both, quark and diquark monomers live on a single site and we placed diquark monomers at the sites $x_{1}, x_{2}$ and $x_{3}$, while quark monomers are placed at $x_{4}$ and $x_{6}$. Dimer terms live on the links and we represent a quark dimer by a single fat line, while diquark dimers are represented by two touching lines on the same link. On the links between $x_{1}$ and $x_{2}, x_{3}$ and $x_{4}$, as well as $x_{4}$ and $x_{5}$ we have placed quark dimers, while on the link between $x_{5}$ and $x_{6}$ we have placed a diquark dimer. Obviously, in our example at every site we have all three color components saturated either by monomer terms or endpoints of dimer terms. We stress that in Fig. 1 the sites simply form a chain, but of course at some site $x$ on a $d$-dimensional lattice one has to take into account all $2 d$ links attached to that site.

In the partition function (3.3) we have to sum over all monomer and dimer assignments that are compatible with a given structure of the complementary domain $\overline{\mathscr{B}}$. We will base this summation 


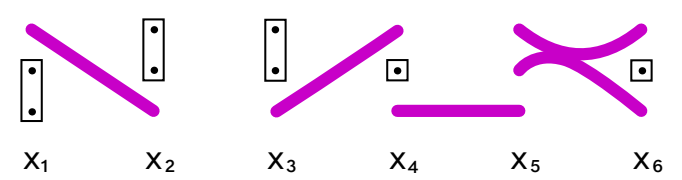

Figure 1: Examples for saturation in the complementary domain.

on our monomer variables $s_{x}=0,1,2$ on the sites and the dimer variables $d_{x, v}=0,1,2$ based on the links. Since at each site $x$ every color has to be occupied by either a monomer or the endpoint of a dimer term, we find the following simple constraint,

$$
s_{x}+\sum_{v}\left[d_{x, v}+d_{x-\hat{v}, v}\right]=3 \quad \forall x \in \overline{\mathscr{B}} .
$$

It turns out that for a given set of fixed monomer variables $s_{x}$ and dimer variables $d_{x, v}$ one can sum over the multiplicities for the different configurations of monomer and dimer arrangements that are compatible with the fixed values of the $s_{x}$ and $d_{x, v}$. We discuss this for the examples in Fig. 1: At site $x_{1}$ we can choose to place the endpoint of the quark dimer at any of the three colors and the remaining two colors are saturated with the diquark monomer. Thus at the site $x_{1}$ we find a degeneracy factor of $F\left(x_{1}\right)=3$. The same analysis holds for the sites $x_{2}$ and $x_{3}$ that both have a degeneracy factor of $F\left(x_{2}\right)=F\left(x_{3}\right)=3$. The situation is different for $x_{4}$ : There we have 3 choices for placing the quark monomer and another 2 choices for placing the endpoints of the two quark dimers, giving a degeneracy of $F\left(x_{4}\right)=6$. For $x_{5}$ and $x_{6}$ we again find a degeneracy of $F\left(x_{5}\right)=F\left(x_{6}\right)=3$, because we have 3 choices for placing the endpoint of the quark dimer at $x_{5}$, respectively the quark monomer at $x_{6}$ and the remaining two colors are saturated by the two endpoints of the diquark dimer. Thus whenever a diquark element is attached at a site $x$, the multiplicity is 3, while it is 6 when only quark elements are used for the saturation at $x$. Thus we obtain $F(x)=6-3 \delta_{s_{x}, 2}-3 \sum_{v}\left[\delta_{d_{x, v}, 2}+\delta_{d_{x-\hat{v}, v}, 2}\right]$ and rewrite the partition sum (3.3) into the form

$Z=\sum_{\{\mathscr{B}\}} \prod_{i} \operatorname{det} D^{(i)} \sum_{\{s, d \in \overline{\mathscr{B}}\}}\left[\prod_{x \in \mathscr{\mathscr { B }}} \delta\left(s_{x}+\sum_{v}\left[d_{x, v}+d_{x-\hat{v}, v}\right]-3\right) F(x)\right](2 m)^{\sum_{x} s_{x}}\left(\frac{1}{3}\right)^{\sum_{x, v}\left[\delta_{d x, v, 1}+\delta_{d x, v, 2}\right]}$.

The first sum is again the sum over all bag configurations and we collect the factors from the corresponding bag determinants. By the second sum $\sum_{\{s, d \in \overline{\mathscr{B}}\}} \equiv \prod_{x \in \overline{\mathscr{B}}} \sum_{s_{x}=0}^{2} \prod_{(x, v) \in \overline{\mathscr{B}}} \sum_{d_{x, v}=0}^{2}$ we denote the sum over all monomer and dimer configurations in the complementary domain. These are subject to the constraints (4.1) at all sites $x$ which we write as a product of Kronecker deltas, here denoted as $\delta(n) \equiv \delta_{n, 0}$. The admissible configurations come with degeneracy factors $F(x)$ at all sites $x$ in the complementary domain, and the weights from the quark mass and the factors $1 / 3$ for quark and diquark dimers. In the form (4.2) the baryon bag representation is better accessible to numerical simulations and we have started to implement the first numerical tests.

\section{The case of strong coupling $\mathrm{SU}(2)$}

We conclude our discussion of bag representations for effective strong coupling degrees of freedom with the case of gauge group SU(2). In this case the effective degrees of freedom are diquarks which are bosons. Thus we expect a different form of the contributions from the bags: 1) The action for 
the bag terms should be bosonic in nature, i.e., we expect to find a Helmholtz operator for the kernel. 2) The bag determinant, which is fully anti-symmetric, should be replaced by a completely symmetric expression. Since the diquarks are nilpotent, we will see that the bag determinant is replaced by a so-called permanent which like the determinant is a sum over all permutations, but the sign of the permutations is omitted.

We start our discussion of SU(2) with the expansion of the nearest neighbor Boltzmann factors as in (2.3) and (2.4). Since for SU(2) we only have two colors, the power series for the Boltzmann factor terminates after the quadratic term. As before, this highest order term is independent of the gauge link, $(\bar{\psi} U \psi)^{2}=2 ! \bar{\psi}_{2} \bar{\psi}_{1} \psi_{1} \psi_{2} \operatorname{det} U \equiv 2 ! \bar{B} B$, where now the baryonic degrees of freedom are diquarks, i.e., $B_{x}=\psi_{x, 1} \psi_{x, 2}, \bar{B}_{x}=\bar{\psi}_{x, 2} \bar{\psi}_{x, 1}$. We find for the nearest neighbor Boltzmann factor

$$
e^{\gamma_{x, v} \bar{\psi}_{x} U_{x, v} \psi_{x+\hat{v}}}=e^{\bar{B}_{x} B_{x+\hat{\nu}}} \sum_{d_{x, v}=0}^{1} \gamma_{x, v}\left(\bar{\psi}_{x} U_{x, v} \psi_{x+\hat{v}}\right)^{d_{x, v}}
$$

In the exponential on the rhs. the staggered sign $\gamma_{x, v}$ has disappeared since it is squared for diquarks. The baryonic/diquark fields $B_{x}$ and $\bar{B}_{x}$ are now made of an even number of Grassmann variables, such that they commute. Combining the nearest neighbor terms (5.1) with the corresponding backward hopping and the mass term we find a bosonic action for the diquark fields

$$
S_{B}[\bar{B}, B]=\sum_{x}\left(4 m^{2} \bar{B}_{x} B_{x}+\sum_{v}\left[\bar{B}_{x} B_{x+\hat{v}}+\bar{B}_{x+\hat{v}} B_{x}\right]\right)=\sum_{x, y} \bar{B}_{x} H_{x, y} B_{x} .
$$

In the last step we have defined the kernel $H_{x, y}$ of the action, which is the sum of a constant term and the discretized Laplace operator, i.e., a discretized Helmholtz operator. Again we write the partition sum as $Z=\int D[\bar{\psi}, \psi] e^{S_{B}[\bar{B}, B]} W[\bar{\psi}, \psi]$, where the weight for the non-baryonic terms is

$$
W[\bar{\psi}, \psi]=\prod_{x} \sum_{s_{x}=0}^{1}\left(2 m \bar{\psi}_{x} \psi_{x}\right)^{s_{x}} \prod_{x, v} \sum_{d_{x, \mu}=0}^{1} \int D[U]\left(-\bar{\psi}_{x} U_{x, v} \psi_{x+\hat{v}} \bar{\psi}_{x+\hat{v}} U_{x, v}^{\dagger} \psi_{x}\right)^{d_{x, v}}
$$

We have already used the fact that only the same powers of $U_{x, v}$ and $U_{x, v}^{\dagger}$ give rise to a singlet and thus a non-trivial strong coupling integral. As a consequence we have the following nontrivial contributions from the non-baryonic terms: Quark monomers for $s_{x}=1$ and quark dimers for $d_{x, v}=1$ (here the corresponding $\mathrm{SU}(2)$ integral [8] gives a factor of 1/2).

Again we can decompose the lattice into bags $\mathscr{B}_{i}$ where we use the baryonic/diquark terms to saturate the Grassmann integral and a complementary domain where we saturate the Grassmann integral with quark monomers and dimers. Note that for SU(2) no diquark terms appear in the complimentary domain - they are the degrees of freedom used inside the bags. Before we can write down the final expression for the partition sum we need to clarify the form of the contribution inside the bags. We already remarked, that for strong coupling $\mathrm{SU}(2)$ the baryon/diquark fields $B_{x}$ and $\bar{B}_{x}$ commute since they are composed of pairs of Grassmann variables. However, they are still nilpotent, such that we obtain the same algebraic form for the integral over the degrees of freedom in the bag, but without the signs that were generated when commuting the SU(3) strong coupling fields. Thus instead of the bag determinant we obtain a so-called permament perm $H^{(i)}$, where $H^{(i)}$ is the Helmholtz operator defined in (5.2) but restricted to the bag $\mathscr{B}_{i}$. The definition of the permanent perm $M$ of a $N \times N$ matrix $M$ reads perm $M=\sum_{\pi(N)} \prod_{k=1}^{N} M_{k, \pi_{k}}$ where the sum 
runs over all permutations of $N$ numbers. The final result for the bag representation for the strong coupling SU(2) case thus reads (the degeneracy factor is always $F(x)=2 \forall x \in \overline{\mathscr{B}}$ )

$$
Z=\sum_{\{\mathscr{B}\}} \prod_{i} \operatorname{perm} H^{(i)} \sum_{\{s, d \in \overline{\mathscr{B}}\}}\left[\prod_{x \in \mathscr{\mathscr { B }}} \delta\left(s_{x}+\sum_{v}\left[d_{x, v}+d_{x-\hat{v}, v}\right]-2\right) F(x)\right](2 m)^{\sum_{x} s_{x}}\left(\frac{1}{2}\right)^{\sum_{x, v} d_{x, v}} .
$$

This example of a case with effective bosonic bag degrees of freedom concludes our presentation of bag techniques for strong coupling gauge fields with fermions.

\section{References}

[1] S. Chandrasekharan, PoS LATTICE 2008 (2008) 003 [arXiv:0810.2419]. P. de Forcrand, PoS LAT 2009 (2009) 010 [arXiv:1005.0539]. U. Wolff, PoS LATTICE 2010 (2010) 020 [arXiv:1009.0657]. C. Gattringer, PoS LATTICE 2013 (2013) 002 [arXiv:1401.7788].

[2] P. Rossi, U. Wolff, Nucl. Phys. B 248 (1984) 105. F. Karsch, K. Mütter, Nucl. Phys. B 313 (1989) 541.

[3] D.H. Adams, S. Chandrasekharan, Nucl. Phys. B 662, 220 (2003) [hep-lat/0303003].

S. Chandrasekharan, F.J. Jiang, Phys. Rev. D 68, 091501 (2003) [hep-lat/0309025].

S. Chandrasekharan, C.G. Strouthos, Phys. Rev. D 69 (2004) 091502 [hep-lat/0401002]. P. de Forcrand, M. Fromm, Phys. Rev. Lett. 104, 112005 (2010) [arXiv:0907.1915]. W. Unger, P. de Forcrand, J. Phys. G 38, 124190 (2011) [arXiv:1107.1553]. P. de Forcrand, S. Kim, W. Unger, JHEP 1302, 051 (2013) [arXiv:1208.2148]. P. de Forcrand et al, Phys. Rev. Lett. 113, 152002 (2014) [arXiv:1406.4397]. W. Unger, PoS LATTICE 2014 (2014) 192 [arXiv:1411.4493]. J. Kim, W. Unger, PoS LATTICE 2016, 035 (2016) [arXiv:1611.09120]. P. de Forcrand, P. Romatschke, W. Unger, H. Vairinhos, PoS LATTICE 2016, 086 (2017) [arXiv:1701.08324]. P. de Forcrand, W. Unger, H. Vairinhos, Phys. Rev. D 97, 034512 (2018) [arXiv:1710.00611].

[4] C. Gattringer, Phys. Rev. D 97, 074506 (2018) [arXiv:1802.09417].

[5] G. Gagliardi, J. Kim, W. Unger, EPJ Web Conf. 175 (2018) 07047 [arXiv:1710.07564]. C. Gattringer, D. Göschl, C. Marchis, EPJ Web Conf. 175, 11007 (2018) [arXiv:1710.08745 ]. O. Borisenko, V. Chelnokov, S. Voloshyn, EPJ Web Conf. 175 (2018) 11021 [arXiv:1712.03064]. C. Marchis, C. Gattringer, Phys. Rev. D 97 (2018) 034508 [arXiv:1712.07546]; Nucl. Phys. B 916 (2017) 627 [arXiv:1609.00124]; PoS LATTICE 2016, 034 (2016) [arXiv:1611.01022]. C. Marchis, "Abelian color cycle and abelian color flux dualization methods for non-abelian lattice field theories," $\mathrm{PhD}$ thesis, University of Graz 2018, arXiv:1809.02415. G. Gagliardi, W. Unger, arXiv:1811.02817.

[6] S. Chandrasekharan, Phys. Rev. D 82, 025007 (2010) [arXiv:0910.5736]. S. Chandrasekharan, Eur. Phys. J. A 49 (2013) 90 [arXiv:1304.4900].

[7] S. Chandrasekharan, A. Li, JHEP 1101, 018 (2011) [arXiv:1008.5146]; Phys. Rev. Lett. 108, 140404 (2012) [arXiv:1111.7204]; Phys. Rev. D 85, 091502 (2012) [arXiv:1202.6572]. S. Chandrasekharan, Phys. Rev. D 86, 021701 (2012) [arXiv:1205.0084]. S. Chandrasekharan, A. Li, Phys. Rev. D 88, 021701 (2013) [arXiv:1304.7761]. E.F. Huffman, S. Chandrasekharan, Phys. Rev. B 89, 111101 (2014) [arXiv:1311.0034]. V. Ayyar, S. Chandrasekharan, Phys. Rev. D 91, 065035 (2015) [arXiv:1410.6474]; Phys. Rev. D 93, 081701 (2016) [arXiv:1511.09071]; JHEP 1610, 058 (2016) [arXiv:1606.06312]. E. Huffman, S. Chandrasekharan, Phys. Rev. D 96, 114502 (2017) [arXiv:1709.03578]. V. Ayyar, S. Chandrasekharan, J. Rantaharju, Phys. Rev. D 97 (2018) 054501 [arXiv:1711.07898].

[8] M. Creutz, J. Math. Phys. 19, 2043 (1978). 